\title{
A Hybrid Approach of Learning and Model-Based Channel Prediction for Communication Relay UAVs in Dynamic Urban Environments
}

\author{
Pawel Ladosz ${ }^{1}$, Hyondong $\mathrm{Oh}^{2}$, Gan Zheng ${ }^{3}$ and Wen-Hua Chen ${ }^{1}$
}

\begin{abstract}
This paper presents the trajectory planning of small UAVs for a communication relay mission in an urban environment. In particular, we focus on predicting the communication strength between air and ground nodes accurately to allow relay UAVs to maximise the communication performance improvement of networked nodes. In urban environments, this prediction is not easily achievable even with good mathematical models as each model is characterized by a series of parameters which are not trivial to obtain or estimate apriori and can vary during the mission. To address the difficulty, this work proposes to integrate a learning-based measurement technique with a probabilistic communication channel model. This hybrid approach is able to predict communication model parameters based on signal strength data which UAVs observe during the mission online, thus achieving better performance compared with the model-based approach in an urban environment. The predicted parameters are based on four discrete urban environment types. Numerical simulations validate the performance and benefit of the proposed approach.
\end{abstract}

Index Terms-Aerial Systems: Applications, Learning and Adaptive Systems, Motion and Path Planning

\section{INTRODUCTION}

$\mathbf{E}$ STABLISHING wireless communication networks is vital in various circumstances such as rescue missions in a natural disaster. In urban environments, wireless communication is limited by the short coverage range and small bandwidth due to line-of-sight (LOS) obstruction by buildings. The following technologies have been used as an attempt to mitigate issues in urban environments: infrastructures (e.g. cell

Manuscript received: September, 8, 2018; Revised December, 12, 2018; Accepted January, 30, 2019.

This paper was recommended for publication by Editor Nancy Amato upon evaluation of the Associate Editor and Reviewers' comments. This work was supported by the UK Engineering and Physical Science Research Council (EPSRC) under the Grant EP/J011525/1, Science and Technology Facilities Council (STFC) under Newton fund with grant number ST/N006852/1, the Basic Science Research Program through the Lockheed Martin Corporation Republic of Korea Science, Technology, Research (RoKST\&R) Initiative, and the 2019 Research Fund (1.190011.01) of UNIST (Ulsan National Institute of Science and Technology).(Corresponding author: Hyondong Oh)

${ }^{1}$ Pawel Ladosz and Wen-Hua Chen are with the Department of Aeronautical and Automotive Engineering, Loughborough University, Loughborough, Leicestershire, LE11 3TU, United Kingdom (Email: \{p.ladosz, w.chen\}elboro.ac.uk).

${ }^{2}$ Hyondong Oh is with School of Mechanical, Aerospace and Nuclear Engineering, Ulsan National Institute of Science and Technology (UNIST), Ulsan, Republic of Korea (Email: h.oh@unist.ac.kr).

${ }^{3}$ Gan Zheng is with Wolfson School of Mechanical, Electrical and Manufacturing Engineering, Loughborough University, Loughborough, Leicestershire, LE11 3TU, United Kingdom (Email: g. zheng(lboro.ac.uk).

Digital Object Identifier (DOI): see top of this page. tower), ground-based radios and satellite communications. The first is often unavailable due to the damage caused by the natural disaster. Besides, in an emergency situation, there is little time to put a temporary infrastructure in place. While the other two do not rely on existing infrastructure, neither of them can solve the issue of obstruction by buildings fully. Furthermore, satellites are limited by the nature of their preplanned orbits. A group of low flying (below 500 meters) and small (less than $7 \mathrm{~kg}$ ) unmanned aerial vehicles (UAVs) can be used as a promising alternative solution since they can be deployed rapidly to the mission area and relocate swiftly to avoid the effect of obstruction by buildings.

Using a group of UAVs for communication relay nodes has its own challenges such as choosing the optimal number of UAVs to cover a given area, developing appropriate communication protocols and planning optimal trajectories considering the dynamic environments. In particular, to plan optimal trajectories, information or prediction on the communication strength between arbitrary points in the air and on the ground becomes quite important. There are several methods to obtain the communication strength between two nodes, which can be generally divided into model-based and measurement-based approaches [1].

Model-based approaches can be subdivided into the range, channel model and probabilistic model-based ones. The rangebased approach such as [2], [3] defines a radius within which communication is assumed to be possible. In the channel model-based approach [4]-[7], the communication strength usually depends on the distance and the existence of direct LOS between nodes. The probabilistic model-based approach, termed as the low-altitude platform model proposed by [8], approximates the probability of LOS occurrence depending on the types of urban environments. The main limitation of model-based approaches is that they need to know a large number of parameters required to compute communication quality between two nodes, which can be challenging to estimate before the mission or online.

Measurement-based approaches were devised to cope with the aforementioned challenge of estimating communication parameters accurately and preferably online. For this, the UAV collects the signal strength data from ground nodes and uses this information to plan its path. They can be largely subdivided into gradient following and learning approaches. In gradient following methods such as [9], the UAV collects the signal strength from ground nodes and calculates the gradient to move towards the optimal position gradually. Gradient 
following methods are unable to cope with non-linearities and discontinuities in the signal strength which are introduced by buildings. Meanwhile, learning-based approaches such as [10], [11] rely on collecting the signal strength data to update a priori communication model using machine learning techniques. Measurement-based approaches were used mainly for stationary environments thus far as the amount of data required for learning and prediction for mobile ground nodes was too high.

To address limitations of aforementioned methods, this work proposes the combination of a learning-based measurement technique with the probabilistic low altitude platform (LAP) model [8] to help plan the UAV relay trajectory. This hybrid approach does not entirely depend on communication parameters known apriori but still can cope with mobile ground nodes (i.e. dynamic environments). Instead of communication parameter estimation, a neural network (NN) is used to predict the discrete urban environment type based on the collected signal strength and elevation angle between air and ground nodes. This predicted discrete urban environment type is used in the LAP model which provides approximated communication quality between any two nodes rapidly. Then, receding horizon-based online trajectory planning is performed to determine optimal control commands for relay UAVs in dynamic environments. To the best of our knowledge, measurementbased approaches (both gradient and learning methods) have rarely been dealt with for dynamic environments due to the amount of data required for learning the entire communication environment or difficulty in computing the smooth gradient. However, in our work, by using the signal strength and elevation angle data to predict the urban type to be used in the LAP model, we are readily able to apply the measurementbased technique (with much reduced data for learning) to dynamic scenarios where ground nodes are moving.

The rest of the paper is organised as follows. The overview of the algorithm and scenarios are presented in Section II. In Section III, the communication channel model and neural network-based channel prediction is described. The receding horizon-based trajectory planner is formulated in Section IV. Section $\mathrm{V}$ shows numerical simulation results to demonstrate the performance of the proposed method, and Section VI presents conclusions and future work.

\section{PROBlem OVERVIEW}

\section{A. Assumptions}

A sample scenario considered in this work is illustrated in Fig. 1. In this scenario, there are a number of mobile ground nodes in an urban environment. UAVs fly in a way to assist ground nodes with their wireless communication equipment for better communication performance.

The assumptions made in this work are stated as follows: i) an urban environment can be modelled as either of the four types: suburban, urban, dense urban and high-rise urban depending on the density and height of buildings; ii) the communication channel model consisting of path loss, transmitted power and shadow fading components is known empirically for each discrete urban environment type, however the discrete

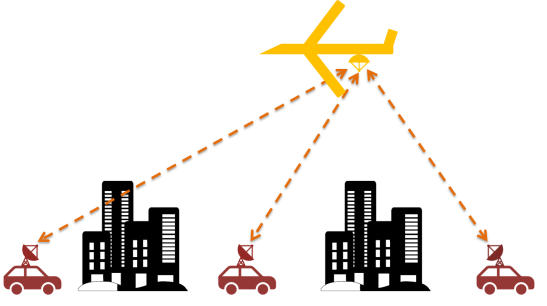

Figure 1. Illustration of the communication relay scenario.

urban environment type for a given scenario is unknown; iii) ground nodes are able to share their current positions with UAVs, but their future paths are unknown to the UAV; and iv) the position and the shape of buildings are unknown.

\section{B. Overview of the Proposed Hybrid Channel Prediction}

Figure 2 shows the overview of the optimal trajectory planning process with the hybrid channel prediction approach. To plan the trajectory of communication relay UAVs, first a cross entropy optimiser (CEO) [12] randomly generates a set of possible trajectories. For each trajectory, the LAP model is used to compute the communication quality of the networked team. To use the correct LAP model, UAVs collect a pair of signal strength and elevation angle between the UAV and ground nodes (hereafter such a pair is called signal strength-angle pairs). With this data, the $\mathrm{NN}$ predictor is used to predict the current discrete urban environment type. Until convergence is reached, the CEO algorithm changes the candidate trajectories and once the convergence criterion is satisfied, the best trajectory is sent to UAVs for execution. Note that, this entire process is periodically performed to cope with the dynamic environment.

\section{LEARNING-BASED COMMUNICATION CHANNEL PREDICTION}

\section{A. Air-to-Ground Channel Modelling}

The communication channel model used in this work is based on the multiple ray tracing simulation with four different types of urban environments: i) Suburban, ii) Urban, iii) Dense urban and iv) High-rise urban [13], [14]. Discrete urban environment types are defined by three layout parameters: $\alpha_{0}$, $\beta_{0}$ and $\gamma_{0}$ and six wireless communication parameters: $k_{1}$, $k_{2}, g_{1}, g_{2}, \mu_{L O S}$ and $\mu_{N L O S}$. Layout parameters are used to establish position, size and height of the buildings, while wireless communication parameters are used to compute the wireless communication strength between arbitrary points in the air and on the ground. Wireless communication parameters for each of the four environment were established in [14] through extensive ray tracing simulations. The parameters for four urban environments are summarised in Table I. Detailed explanations on each parameter group are presented below.

The layout parameters are defined as: $\alpha_{0}$ is the ratio of the built-up land area to the total land area, $\beta_{0}$ is the mean number of buildings per unit area (buildings $/ \mathrm{km}^{2}$ ) and $\gamma_{0}$ is a height parameter defining the scale parameter of the Rayleigh distribution so that the $k$-th building has a height of $h_{k} \sim$ 


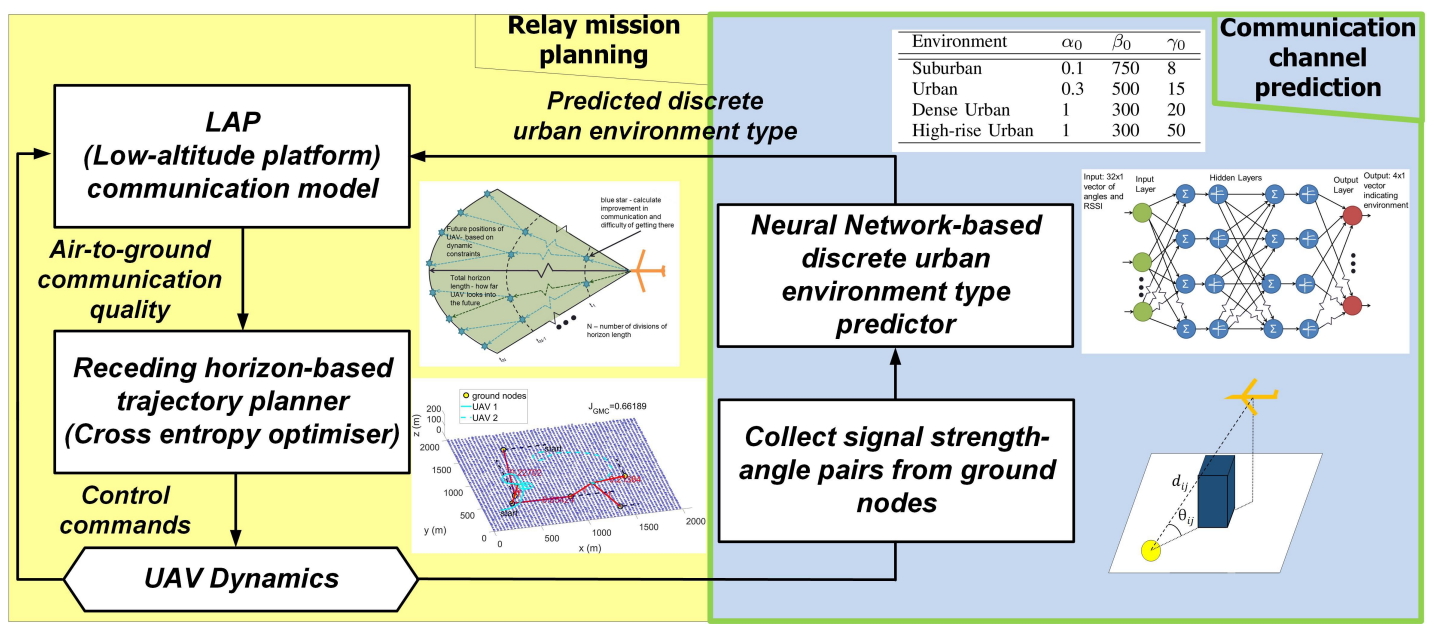

Figure 2. Overview of the optimal trajectory planning process for communication relay UAVs.

$\mathcal{R}\left(\gamma_{0}\right)$, where $k \in\left(1,2 \ldots n_{z}\right)$ and $n_{z}$ is the total number of buildings per scenario. In this study, a standard city layout [14] is used as shown in Fig. 3. The benefit of using this city model is easy generation of multiple cities with different heights for Monte Carlo simulations. With the above city

Table I

DIFFERENT CITY ENVIRONMENT LAYOUT AND COMMUNICATION PARAMETERS

\begin{tabular}{lllll}
\hline Environment & $\left(\alpha_{0}, \beta_{0}, \gamma_{0}\right)$ & $\left(k_{1}, k_{2}\right)$ & $\left(g_{1}, g_{2}\right)$ & $\left(\mu_{L O S}, \mu_{N L O S}\right)$ \\
\hline \hline Suburban & $(0.1,750,8)$ & $(11.25,0.06)$ & $(32.17,0.03)$ & $(0.1,21)$ \\
Urban & $(0.3,500,15)$ & $(10.39,0.05)$ & $(29.6,0.03)$ & $(1,20)$ \\
Dense Urban & $(1,300,20)$ & $(8.96,0.04)$ & $(35.97,0.04)$ & $(1.6,23)$ \\
High-rise Urban & $(1,300,50)$ & $(7.37,0.03)$ & $(37.08,0.03)$ & $(2.3,34)$ \\
\hline
\end{tabular}

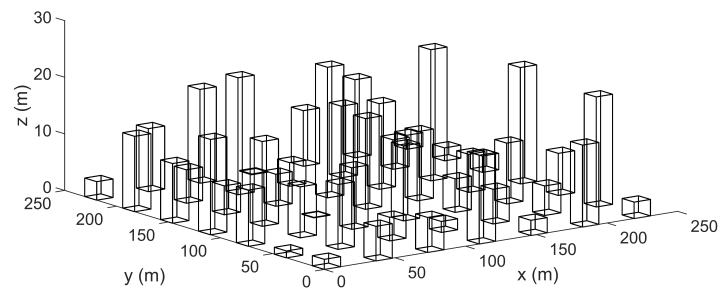

Figure 3. A sample city generated with parameters: $\alpha_{0}=0.1, \beta_{0}=750$ and $\gamma_{0}=8$.

model, the communication channel model can be defined as [15]:

$$
P_{r, i j}=P_{t, j}-L_{d B, i j}-\Psi_{i j}
$$

where $P_{r, i j}(\mathrm{dBm})$ is the received signal power strength of node $i$ from node $j . P_{t, j}(\mathrm{dBm})$ is the transmitted power by node $j, L_{d B, i j}$ represents the free space path loss between nodes $i$ and $j$, and $\Psi_{i j}$ is the shadow fading component accounting for diffraction and multipath fading. Note that, for simplicity, $P_{t, j}$ is assumed to be the same for all nodes in this work. $\Psi_{i j}$ is assumed to be a Gaussian random variable defined as $\Psi_{i j} \sim \mathcal{N}\left(\mu_{i j}, \sigma_{i j}^{2}\right)$, where $\mu_{i j}$ and $\sigma_{i j}^{2}$ are the mean and variance parameters. $L_{d B, i j}$ can be represented as:

$$
L_{d B, i j}=10 \alpha \log _{10}\left(\frac{4 \pi f_{c} d_{i j}}{c}\right),
$$

where $f_{c}$ is the central frequency, $\alpha$ is the path loss exponent, $d_{i j}$ is the distance between nodes $i$ and $j$, and $c$ is the speed of light. Note that $P_{r, i j}$ follows the Gaussian distribution and can be expressed as:

$$
P_{r, i j} \sim \mathcal{N}\left(P_{t, j}-L_{d B, i j}-\mu_{i j}, \sigma_{i j}^{2}\right) .
$$

To be more specific, the shadow fading $\Psi_{i j}$ can take either of two distributions: $\Psi_{L O S, i j} \sim \mathcal{N}\left(\mu_{L O S, i j}, \sigma_{L O S, i j}^{2}\right)$ and $\Psi_{N L O S, i j} \sim \mathcal{N}\left(\mu_{N L O S, i j}, \sigma_{N L O S, i j}^{2}\right)$ for cases of line-ofsight (LOS) and non-LOS (NLOS), respectively. In this work, $\mu_{L O S, i j}$ and $\mu_{N L O S, i j}$ are assumed to be known and constant for a given discrete urban environment type, whereas $\sigma_{L O S, i j}^{2}$ and $\sigma_{N L O S, i j}^{2}$ can be modelled as:

$$
\begin{gathered}
\sigma_{L O S}^{2}=k_{1} \exp \left(k_{2} \theta_{i j}\right), \quad \text { and } \\
\sigma_{N L O S}^{2}=g_{1} \exp \left(g_{2} \theta_{i j}\right),
\end{gathered}
$$

where $\theta_{i j}$ is the elevation angle of the UAV with respect to the ground. Communication parameters $k_{1}, k_{2}, g_{1}, g_{2}$, $\mu_{L O S}$ and $\mu_{N L O S}$ are summarised in Table I. It is worthwhile noting that for more realistic urban environments other than a standard city layout with a squared area for all the buildings as shown in Fig. 3, further studies would be required in the field of communication modelling; however, the general trend for the probability of LOS and NLOS occurrence and corresponding communication quality would still be similar as $\alpha, \beta$ and $\gamma$ contains the necessary information of a certain urban environment.

\section{B. LAP Communication Model}

To allow the UAV to predict the communication strength of ground nodes during trajectory planning, the choice between $\Psi_{L O S, i j}$ and $\Psi_{N L O S, i j}$ needs to be made. For this purpose, we use the method from [8], [13], which predicts the probability 
of LOS (or NLOS) occurrence based on the elevation angle and discrete urban environment type specified by $\alpha_{0}, \beta_{0}$ and $\gamma_{0}$. In this approach, the LOS probability between two nodes can be determined as:

$$
P\left(L O S, \theta_{i j}\right)=\frac{1}{1+\bar{a} \exp \left(-\bar{b}\left[\theta_{i j}-\bar{a}\right]\right)},
$$

where $\theta_{i j}$ is the elevation angle between the air and ground nodes and $\bar{a}$ and $\bar{b}$ are parameters of the S-curve dependent on $\alpha_{0}, \beta_{0}$ and $\gamma_{0}$ [8]. Then, the prediction of $\Psi$ can be made by defining the LOS probability threshold $P_{t}$ as:

$$
\Psi_{i j}= \begin{cases}\Psi_{L O S, i j}, & \text { if } P\left(L O S, \theta_{i j}\right)>P_{t}, \\ \Psi_{N L O S, i j}, & \text { otherwise. }\end{cases}
$$

\section{Learning-Based Channel Prediction}

The signal strength of ground nodes with $P\left(L O S, \theta_{i j}\right)$ in Eq. (6) obtained from the UAV depends on the urban environment parameters. When the urban parameters are unknown, the learning-based approach could be utilised with the collected signal strength data to predict the urban environment parameters. To this end, this study adopts a feedforward neural network (NN) classifier with two hidden layers and 10 neurons in first hidden layer as depicted in Fig. 4. Classification is necessary as communication parameters are defined for four environments listed in Table I. The input consists of 16 pairs of signal strength and elevation angles and the output is a vector $O \in \mathbb{R}^{1 \times 4}$ where each element is responsible for the probability of being in one of four environments which lies between 0 and 1 . To be more specific, the output is defined as $\left[\begin{array}{llll}0 & 0 & 0 & 1\end{array}\right]$ for the suburban environment, $\left[\begin{array}{llll}0 & 1 & 0 & 0\end{array}\right]$ for the urban environment, $\left[\begin{array}{llll}0 & 0 & 1 & 0\end{array}\right]$ for the dense urban environment and $\left[\begin{array}{llll}0 & 0 & 0 & 1\end{array}\right]$ for the high-rise urban environment. Note that, during the trajectory planning process, the urban-type prediction by the $\mathrm{NN}$ is periodically performed with the most recently collected data for a certain period of time. In case of multiple UAVs, each UAV is assumed to be able to make its own prediction based on the signal data it has obtained and then shares its prediction with the rest of the group for improving the prediction accuracy.

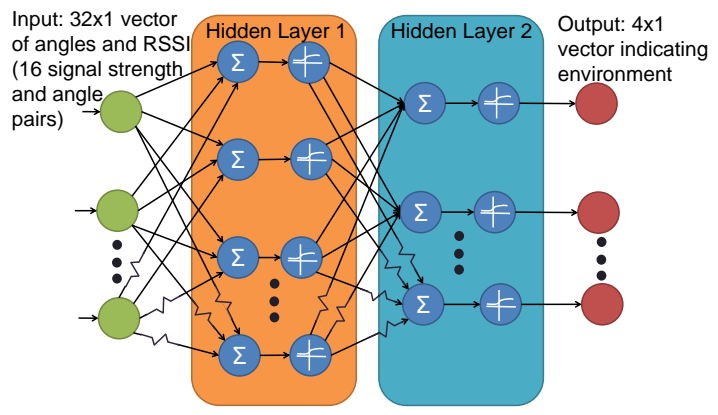

Figure 4. The neural network schematic showing parts of the used network. Sigmoid is used as the output activation function.

The NN was trained using the scaled conjugate backpropagation algorithm with a cross-entropy error function on 12 randomly-generated scenarios (3 scenarios per each urban environment) with 12 stationary ground vehicles. The UAV performed the back and forth search pattern and collected 26,064 signal strength and elevation angle pairs overall.

Before considering other aspects of the NN, it is important to address the appropriate number of inputs to the NN. In general, the more pairs are used for prediction, the better the output is. However, more data will require longer collection time, which increases time of initial prediction and the frequency of consecutive predictions. To determine how many signal strength-angle pairs are sufficient, we tested 4, 8, 16, 32 and 64 pairs and compared the prediction accuracy and computation time averaged over a hundred runs. Results of these tests are summarised in Table II. It can be seen that from 4 to 16 signal strength-angle pairs, the urban type prediction accuracy increases steadily by about $10 \%$ for each increase in data points, while between 32 and 64 pairs the increase is much smaller (by $3 \%$ only). Thus, 16 is chosen as a tradeoff between the accuracy and the prediction speed

Table II

\begin{tabular}{|c|c|c|}
\hline $\begin{array}{l}\text { Number of angle- } \\
\text { RSSI pairs }\end{array}$ & Accuracy & $\begin{array}{l}\text { Computation time (s) averaged } \\
\text { over } 3000 \text { predictions }\end{array}$ \\
\hline 4 & $50.1 \%$ & $3.6 \times 10^{-5}$ \\
\hline 8 & $60.8 \%$ & $3.9 \times 10^{-5}$ \\
\hline 16 & $70.9 \%$ & $9.5 \times 10^{-5}$ \\
\hline 32 & $73 \%$ & $2.6 \times 10^{-4}$ \\
\hline 64 & $76 \%$ & $3.6 \times 10^{-4}$ \\
\hline
\end{tabular}

PREDICTION QUALITY WITH DIFFERENT SIGNAL STRENGTH-ANGLE PAIR

To investigate the quality of the $\mathrm{NN}$ training, a confusion matrix is used (Fig. 5). In the matrix, diagonal terms (in green) are the number of the correctly classified discrete urban environment type along with the percentage of total sample. The red off diagonal elements are the number of target environments incorrectly classified. The edges of the matrix in grey are the total percentage of the correctly classified (green) and incorrectly classified (red) discrete urban environment type. This figure shows that majority of errors occur between neighbouring classes which are next to each other.

The proposed NN is compared against other machine learning techniques: $\mathrm{k}$-nearest neighbour (with the Minkowski distance metric and equal distance weight), support vector machine (with a linear kernal function, automatic scale mode and one-vs-one multiclass method) and decision trees (with 100 maximum splits and Gini's diversity index for split criterion). All approaches are trained on the same data set. Table III indicates that the $\mathrm{NN}$ is at least about $9 \%$ more accurate than other learning methods with less computational burden. More advanced deep learning techniques (e.g. convolutional NN or recurrent $\mathrm{NN}$ ) could readily be applied to improve the accuracy further.

Currently, the $\mathrm{NN}$ is limited to predicting one of the four discrete urban environment types. This is because, in the current LAP communication model [8], only four urban environments were considered. Subsequently, communication parameters are available only for four discrete urban environment types. To compute communication parameter values for arbitrary environments, more fundamental studies would 

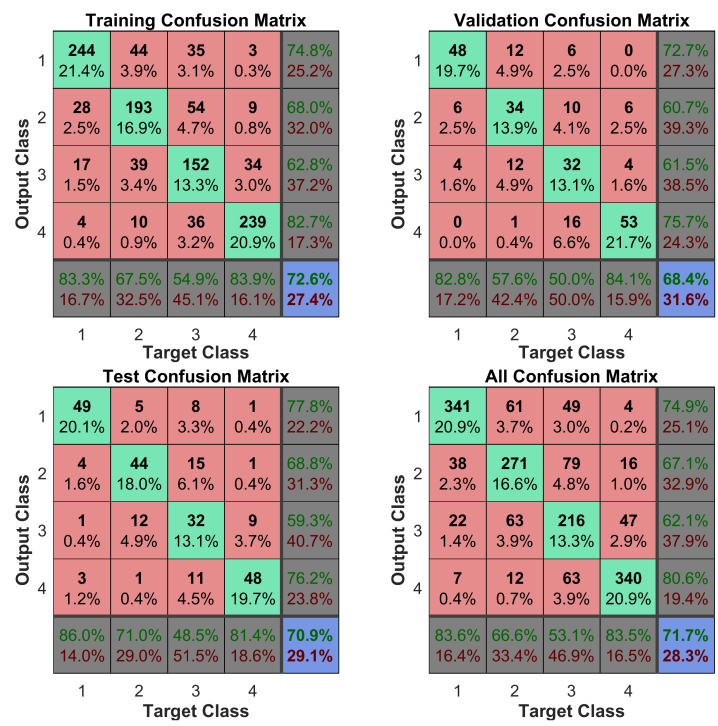

Figure 5. Confusion matrices for the Neural Network with 16 angle-strength pairs. Overall accuracy for test cases is $70.9 \%$.

Table III

ACCURACY OF DIFFERENT LEARNING METHODS

\begin{tabular}{llll}
\hline Method & Accuracy & $\begin{array}{l}\text { Computation time (s) averaged } \\
\text { over 3000 predictions }\end{array}$ \\
\hline \hline NN & $70.9 \%$ & $9.5 \times 10^{-5}$ & \\
$\begin{array}{l}\text { support vector } \\
\text { machine }\end{array}$ & $62.2 \%$ & $6.2 \times 10^{-3}$ & \\
$\begin{array}{l}\text { k-nearest neigh- } \\
\text { bours }\end{array}$ & $61.9 \%$ & $6.9 \times 10^{-3}$ & \\
Decision tree & $46 \%$ & $3.6 \times 10^{-4}$ & \\
\hline
\end{tabular}

need to be performed in the level of communication modelling, which is beyond the scope of this work.

\section{Communication Performance Metrics}

To define the performance metric to plan an optimal trajectory for the relay UAV, two steps are performed: i) conversion of $P_{r, i j}$ to the probability of successful communication and ii) computation of the global message connectivity (GMC) [5] to represent how much the UAV improves the communication performance of the networked group.

To define the probability of successful communication, the signal to noise ratio (SNR) are defined by subtracting the noise power $P_{n, i j}$ from the mean received power strength $P_{r, i j}$ in Eq. (3) as:

$$
\Gamma_{i j} \sim \mathcal{N}\left(\mu_{\gamma, i j}, \sigma_{i j}^{2}\right)
$$

where $\mu_{\gamma, i j}=P_{t, i j}-L_{d B, i j}-\mu_{i j}-P_{n, i j}$ and $P_{n, i j}$ is a noise power $(\mathrm{dBm})$ calculated as:

$$
P_{n, i j}=10 \log _{10}\left(K T B_{i j}\right)+30,
$$

where $K$ is the Boltzman constant, $T$ is ambient temperature, and $B_{i j}$ is the bandwidth. Following steps from [16], the probability of successful communication is then expressed as:

$$
P_{s, i j}\left(\Gamma_{i j} \geq \gamma\right)=Q\left(\frac{\mu_{\gamma, i j}-\gamma}{\sigma_{i j}}\right)
$$

where $\gamma$ is the required minimum SNR defined by the user and $Q$ is the complementary error function.

To measure improvement of communication among a group of ground nodes, the concept of the GMC is used. Before formally defining the GMC, the minimum spanning tree (MST) needs to be introduced. The MST is defined as a subset of a graph where all nodes are connected to each other but there are no loops, having a minimum (or at least the same as the minimum, as there can be several minimum spanning trees in a single graph) sum of edge weights [17]. In this work, the MST is calculated among the UAV and ground nodes using the Kruskal algorithm [18]. The MST allows us to cope with a large group of air and ground nodes, where the number of direct connection increases significantly, as it reduces the number of connections significantly. With the MST defined, the GMC can be formally defined as the probability of a message being successfully transmitted to all nodes within the MST [5].

With the knowledge of the positions of UAVs and ground nodes, the GMC can be computed as follows. Let us define $A^{\prime} \in \mathbb{R}^{(n+m) \times(n+m)}$ as an adjacency matrix, where $n$ is the number of UAVs and $m$ is the number of ground vehicles. The adjacency matrix defines which connections are within the MST, and thus, $A_{i j}^{\prime}=1$ if the link from node $i$ to node $j$ is the part of the MST, and $A_{i j}^{\prime}=0$, otherwise. With the adjacency matrix, the GMC communication performance $J_{G M C}$ can be calculated as the sum of the probability of successful communication of all connections within the MST as:

$$
J_{G M C}\left(\overline{\mathbf{x}}^{\text {pos }}, \overline{\mathbf{x}}^{g, p o s}\right)=\frac{\sum_{i=1}^{n+m} \sum_{j=1}^{n+m} A_{i j}^{\prime} P_{s, i j}}{m+n}
$$

where $\overline{\mathbf{x}}^{\text {pos }} \in \mathbb{R}^{3 \times n}$ and $\overline{\mathbf{x}}^{g, p o s} \in \mathbb{R}^{3 \times m}$ represent the position of UAVs and ground nodes, respectively.

It is worth noting that some other performance metrics could also be used such as the worst case connectivity or modified GMC [6].

\section{RECEDing Horizon-BAsed OnLINe TRAJeCtory PLANNING}

Receding horizon-based trajectory planning is used to determine the optimal path for the relay UAV considering the situation where ground nodes move. The receding horizon technique is based on the concept of the time horizon and prediction based on the known dynamic/kinematic model. The time horizon is used to determine how far into the future the prediction of the UAV path is made. To reduce the computational burden, the time horizon is discretised into $N$ steps, each with a pre-specified length. At each step, improvement in communication and corresponding UAV control commands are computed. The best path is determined by the most optimal combination of control inputs. To account for situation changes through the scenario, only control input from the first horizon step is taken, and the procedure is repeated. 


\section{A. UAV Model}

To use the receding horizon-based planner, the UAV dynamics is modelled. In this work, a simple dynamic model is used, which can be represented as [19]:

$$
\left(\begin{array}{c}
\dot{x} \\
\dot{y} \\
\dot{\psi} \\
\dot{v} \\
\dot{\omega}
\end{array}\right)=f(\mathbf{x}, \mathbf{u})=\left(\begin{array}{c}
v \cos \psi \\
v \sin \psi \\
\omega \\
-\frac{1}{\tau_{v}} v+\frac{1}{\tau_{v}} u_{v} \\
-\frac{1}{\tau_{\omega}} \omega+\frac{1}{\tau_{\omega}} u_{\omega}
\end{array}\right)
$$

where $\mathbf{x}=\left(\begin{array}{lllll}x & y & \psi & v & \omega\end{array}\right)^{T}$ are the inertial position, heading, speed and yaw rate of the UAV, respectively. $\tau_{v}$ and $\tau_{\omega}$ are time constants accounting for the actuator response delay, which can be determined experimentally for a given UAV model. $u_{\omega}$ and $u_{v}$ are command inputs in the form of the turning rate and velocity, respectively. $v$ is constrained by the maximum and minimum velocity $v_{\min }$ and $v_{\max }$, and $\omega$ is constrained by the maximum and minimum heading rate $\omega_{\text {min }}$ and $\omega_{\max }$ due to physical limitations of the fixed-wing UAV. To be able to use the UAV model in the receding horizon-based framework, Eq. (12) is discretised using Euler integration as:

$$
\mathbf{x}_{k+1}=f_{d}\left(\mathbf{x}_{k}, \mathbf{u}_{k}\right)=\mathbf{x}_{k}+T_{s} f\left(\mathbf{x}_{k}, \mathbf{u}_{k}\right)
$$

where $\mathbf{x}_{k}=\left(\begin{array}{lllll}x_{k} & y_{k} & \psi_{k} & v_{k} & \omega_{k}\end{array}\right)^{T}$ and $T_{s}$ is a sampling time.

For the UAV to be able to predict the communication performance at each possible position in the receding horizon length, it needs to predict where ground nodes will be at that time the UAV reaches that position. Generally, current ground vehicles positions are known, but their future paths and plans need to be predicted. For this prediction, the Kalman filter from [6], [20] is employed in this work.

\section{B. Receding Horizon-Based Planning Formulation}

Receding horizon-based trajectory planning is formulated to find the optimal set of command inputs $U^{i}=$ $\left(U_{v}^{i}, U_{\omega}^{i}\right)^{T}$ where $U_{v}^{i}=\left(u_{v, 0}^{i}, u_{v, 1}^{i}, \ldots, u_{v, N-1}^{i}\right), U_{\omega}^{i}=$ $\left(u_{\omega, 0}^{i}, u_{\omega, 1}^{i}, \ldots, u_{\omega, N-1}^{i}\right)$ for $i$-th UAV, which minimizes the following performance index $J$ :

$$
\begin{aligned}
& J \triangleq \Phi\left(\overline{\mathbf{x}}_{N}, \overline{\mathbf{x}}_{N}^{g}\right)+ \sum_{k=0}^{N-1} L\left(\overline{\mathbf{x}}_{k}, \overline{\mathbf{x}}_{k}^{g}, u_{\omega, k}^{i}, u_{v, k}^{i}\right) \\
& \text { s.t. } \quad \mathbf{x}_{k+1}^{i}=f_{d}\left(\mathbf{x}_{k}^{i}, u_{\omega, k}^{i}, u_{v, k}^{i}\right), \\
& \omega_{\min } \leq u_{\omega, k}^{i} \leq \omega_{\max }, \\
&\left|u_{\omega, k}^{i}-u_{\omega, k-1}^{i}\right| \in\left\{0, \Delta u_{\omega}\right\} \\
& v_{\min } \leq u_{v, k}^{i} \leq v_{\max }, \\
&\left|u_{v, k}^{i}-u_{v, k-1}^{i}\right| \in\left\{0, \Delta u_{v}\right\},
\end{aligned}
$$

where:

$$
\begin{gathered}
\Phi\left(\overline{\mathbf{x}}_{N}, \overline{\mathbf{x}}_{N}^{g}\right) \triangleq p_{c} \frac{1}{J_{G M C}\left(\overline{\mathbf{x}}_{N}^{\text {pos }}, \overline{\mathbf{x}}_{N}^{g, p o s}\right)}, \\
L\left(\overline{\mathbf{x}}_{N}, \overline{\mathbf{x}}_{N}^{g}, u_{\omega, k}^{i}, u_{v, k}^{i}\right) \triangleq \frac{1}{2}\left[q_{c} \frac{1}{J_{G M C}\left(\overline{\mathbf{x}}_{k}^{\text {pos }}, \overline{\mathbf{x}}_{k}^{g, p o s}\right)}+\right. \\
\left.+r_{\omega}\left(\frac{u_{\omega, k}^{i}}{\omega_{\max }}\right)+r_{v}\left(\frac{u_{v, k}^{i}}{v_{\max }}\right)\right]^{2}
\end{gathered}
$$

where $J_{G M C}$ represents the GMC performance index as defined in Eq. (11) and $\overline{\mathbf{x}}_{k}^{\text {pos }}$ and $\overline{\mathbf{x}}_{k}^{g, p o s}$ are the position of UAVs and ground nodes at the $k$-th time, respectively. $p_{c}, q_{c}$ and $r_{\omega}$ are constant weighting factors. The control sequence $U^{i}$ which optimises the performance index is found by using the CE optimisation algorithm [12]. The first term in Eq. (14), $\Phi\left(\overline{\mathbf{x}}_{N}, \overline{\mathbf{x}}_{N}^{g}\right)$ is the communication improvement by the UAV at the final position given the UAV follows the path defined by a control sequence. The second term $\sum_{k=0}^{N-1} L\left(\bar{x}_{k}, \bar{x}_{k}^{g}, u_{\omega, k}, u_{v, k}\right)$ is used to account for the communication performance improvement and the cost of moving from one position to the other (i.e. related with control efforts or energy as shown in Eq. (21)) in the receding horizon length. Eqs. (15) (19) represent constraints of relay UAVs for this problem. The first constraint is used to impose the UAV dynamic model defined in Eq. (13) in finding the optimal control command. The second one limits the maximum turning rate for the UAV, and the third one discretises the problem by ensuring that the next command is different from the previous one by $\left\{0, \Delta u_{\omega}\right\}$. The next two are similar but applied to the velocity command. Note that, for multiple UAVs, the path is planned once at a time for each UAV (i.e. sequentially and locally), while others are kept fixed. Negotiation/cooperation of the optimal set of command inputs between UAVs until converged to the global optimal solution for the entire UAV group by considering the effect of trajectory change of other UAVs would improve the performance, but this issue remains as future work.

\section{Numerical Simulation Results}

In this section, the performance of the proposed approach is investigated through a set of 48 Monte Carlo simulations. Parameters of simulations and wireless communication are given in Table $\mathrm{V}$ and IV, respectively. The proposed approach, receding horizon $(\mathrm{RH})$-based trajectory planning with the hybrid channel prediction (termed as $\mathrm{RH}+\mathrm{NN}$ with the LAP model), is compared with three other approaches as summarised below.

- RH with known map - an approach where building sizes, positions and effects on the communication quality are fully known. It allows the UAV to make the perfect prediction of the communication performance between arbitrary points. Such knowledge is unlikely to be available in a real world scenario, thus this approach serves as a benchmark only. More details about this approach can be found in [6]

- RH with known LAP - similar to the proposed approach in that it uses Eq. (6) to predict the probability of being in LOS, but $\alpha_{0}, \beta_{0}$ and $\gamma_{0}$ are assumed to be known a priori. This approach is not expected to perform as well as RH with the known map due to the probabilistic prediction of LOS occurrence compared to the exact LOS computation of RH with known map approach.

- RH with incorrect LAP - an approach where incorrect $\alpha_{0}, \beta_{0}$ and $\gamma_{0}$ are used for RH with the known LAP. This approach is intended to show what happens when incorrect prediction about the urban environment type is made. 
Note that for the MST construction during the prediction stage, connections between ground nodes are assumed to be in NLOS at all times, while connections between UAVs are assumed to be in LOS at all times.

Table IV

COMMUNICATION PARAMETERS

\begin{tabular}{lll}
\hline Parameter & Value & Unit \\
\hline \hline Transmission power $\left(P_{t}\right)$ & 40 & $\mathrm{dBm}$ \\
Frequency $\left(f_{c}\right)$ & 2.0 & $\mathrm{GHz}$ \\
Attenuation factor $(\alpha)$ & 2.5 & $\mathrm{n} / \mathrm{a}$ \\
Communication Properties & $(11.25,0.06,32.17,0.03)$ & $\mathrm{n} / \mathrm{a}$ \\
$\left(k_{1}, k_{2}, g_{1}, g_{2}\right)$ & & \\
mean LOS fading $\left(\mu_{L O S}\right)$ & 0.1 & $\mathrm{n} / \mathrm{a}$ \\
mean NLOS fading & 21 & $\mathrm{n} / \mathrm{a}$ \\
$\left(\mu_{N L O S}\right)$ & & $\mathrm{MHz}$ \\
Bandwidth $\left(B_{i j}\right)$ & 5 & \\
\hline
\end{tabular}

Table V

SIMULATION PARAMETERS

\begin{tabular}{lll}
\hline Parameter & Value & Unit \\
\hline \hline Actuator delay $\left(\tau_{\omega}, \tau_{v}\right)$ & $(1 / 3,1 / 3)$ & $\mathrm{sec}$ \\
speed constraints $\left(v_{\min }, v_{\max }\right)$ & $(10,30)$ & $\mathrm{m} / \mathrm{s}$ \\
Heading rate constraint $\left(\omega_{\min }, \omega_{\max }\right)$ & $(-0.4,0.4)$ & $\mathrm{rad} / \mathrm{s}$ \\
Receding horizon step $(N)$ & 5 & $\mathrm{~N} / \mathrm{A}$ \\
Horizon steps & $(0.5,4.5,5,5,5)$ & $\mathrm{sec}$ \\
Maximum heading rate change $\left(\Delta u_{\omega}\right)$ & 0.1 & $\mathrm{rad} / \mathrm{s}$ \\
Maximum velocity change $\left(\Delta u_{v}\right)$ & 5 & $\mathrm{~m} / \mathrm{s}$ \\
Weighting factors $\left(p_{c}, q_{c}, r_{\omega}\right)$ & $\left(-1000, p_{c} / N, 1\right) \mathrm{N} / \mathrm{A}$ \\
Urban Environment Parameters $\left(\alpha_{0}\right.$, & $(0.1,750,8)$ & $\mathrm{N} / \mathrm{A}$ \\
$\left.\beta_{0}, \gamma_{0}\right)$ & & \\
Urban Environment Parameters $\left(\alpha_{0}\right.$, & $(1,300,20)$ & $\mathrm{N} / \mathrm{A}$ \\
$\left.\beta_{0}, \gamma_{0}\right)$ for RH with incorrect LAP & & \\
model & & $K$ \\
$\begin{array}{l}\text { Ambient temperature }(T) \\
\text { Number of UAVs }(n)\end{array}$ & 293 & $\mathrm{~K} / \mathrm{A}$ \\
number of ground nodes $(m)$ & 6 & N/A \\
\hline
\end{tabular}

Figure 6 shows a sample scenario from Monte Carlo simulations with trajectories using aforementioned four methods. In this scenario, issues with RH with the incorrect LAP model (i.e. using incorrectly-guessed parameters) approach are apparent. Within the time frame of the scenario, other three approaches send one UAV to around $(x, y)=(1400,500)$ and the other to $(x, y)=(400,700)$ to serve as relay nodes there. However, RH with the incorrect LAP model makes both UAVs to stay around the $(x, y)=(400,700)$ where they can only help three ground nodes. It is likely that RH with the incorrect LAP computed that there is no viable position where the relay UAV could help by flying to other position. Such an erroneous decision is made as RH with the incorrect LAP uses a city with buildings which are much higher and bigger than they actually are.

Figure 7 shows the comparison of the performance among four approaches with parameters from Table V. It can be seen that RH with the known map has the best performance. Notably, the RH+NN approach has the probability of successful communication not much worse than RH with the known LAP model. The proposed approach is significantly better than RH with the incorrect LAP model by about $10 \%$ increased probability of achieving the desired communication strength.

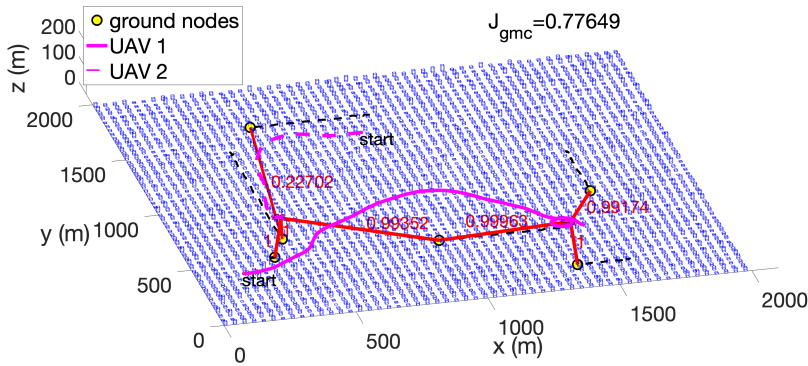

(a) RH with the known map

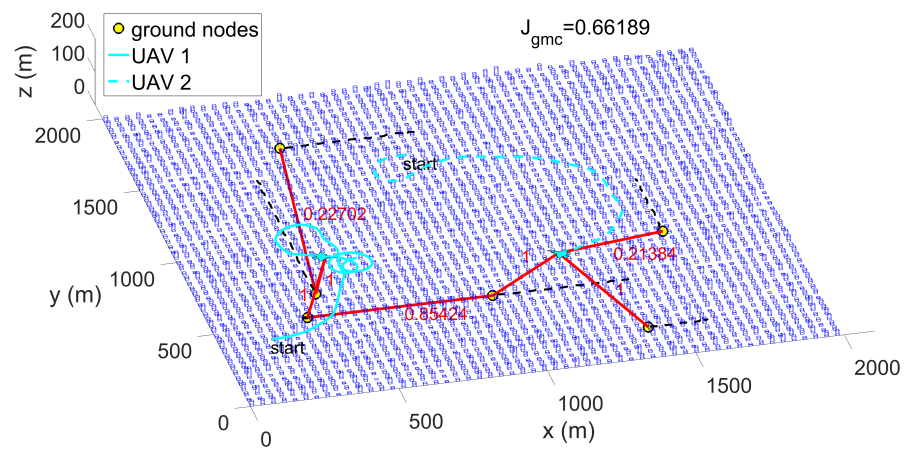

(b) $\mathrm{RH}+\mathrm{NN}$ with LAP model

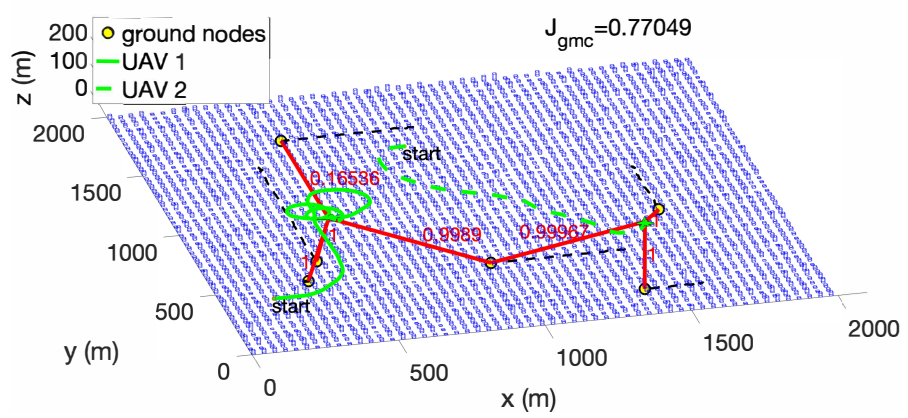

(c) RH with the known LAP model

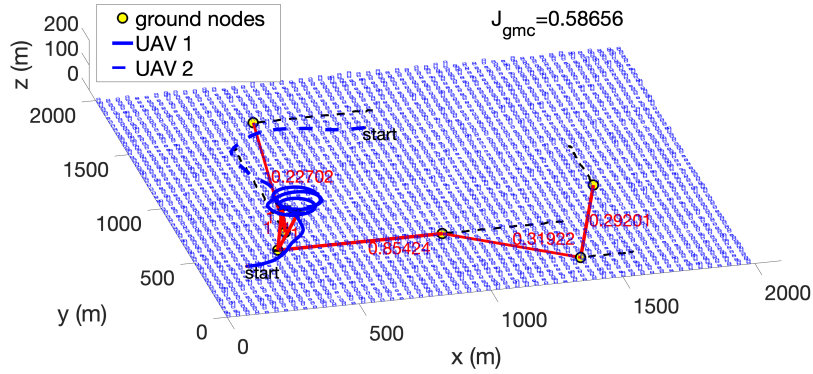

(d) RH with the incorrect LAP model

Figure 6. Simulation results using different approaches. The red lines represent the MST with the corresponding probability of successful communication.

It is worth noting that the probability shown here is averaged over all connections within the MST.

The quality of the discrete urban environment type prediction using the $\mathrm{NN}$ is summarised as follows. 41 times out of 48 cases, the NN made the correct prediction of the discrete urban environment type at the end of the simulations, while 7 


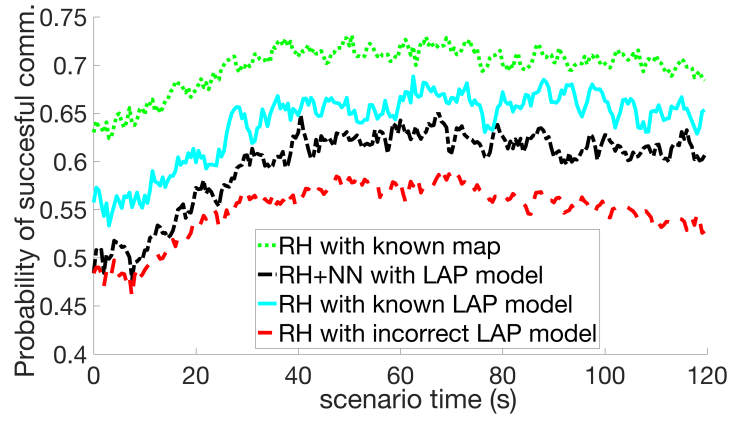

Figure 7. Performance comparison of the proposed method with others, averaged over 48 Monte Carlo simulations.

cases were incorrectly predicted. It took 44.5 seconds (of the scenario time) for the NN to converge to the constant value on average (i.e. after 44.5 seconds, the $\mathrm{NN}$ would rarely change its prediction) while the minimum convergence time was 3.5 seconds. The incorrect predictions result from the difference between $\mathrm{RH}+\mathrm{NN}$ with LAP model and RH with known LAP model. Even with the slightly wrong prediction, the trajectory planner is still able to provide a reasonably good performance as shown in Fig. 7.

Finally, the performance with the increasing number of UAVs is investigated in Fig. 8 where the results are obtained by averaging from the final 20 seconds of 48 Monte Carlo simulations. Each of the 48 simulations was performed with the different number of UAVs and parameters from Table V. No UAV case indicates the scenario with just ground nodes for the comparison purpose. With no communication relay UAV in the area, there is a very low probability of establishing a successful communication for the networked nodes of only $30 \%$. As the number of UAVs increases, the probability of successful communication increases.

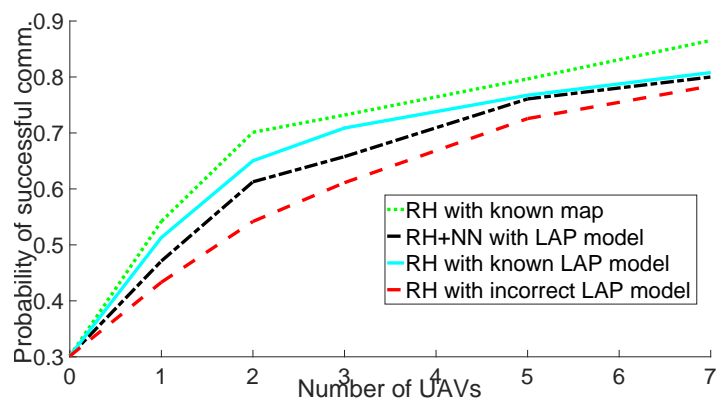

Figure 8. The averaged performance over 48 scenarios for the final 20 seconds of the flight with the different number of UAVs.

\section{CONCLUSiOnS AND Future WORK}

In this work, a hybrid channel modelling approach in support of the trajectory planning for communication relay UAVs was studied. It was shown that the proposed NN-based approach can predict the right discrete urban environment type that provides essential information to be used in the LAP model. This approach is useful as it requires very little knowledge about the mission area, yet it can be implemented quickly with the improved performance. In the future, a simple two-layer neural network could be replaced with a deep learning technique such as the recurrent neural network approach which efficiently deals with time series data. Besides, experiments using ground robots and UAVs will be performed to validate the benefit of the proposed approach.

\section{REFERENCES}

[1] H.-S. Shin and P. Segui-Gasco, "UAV swarms: decision-making paradigms," Encyclopedia of Aerospace Engineering, pp. 1-13, 2014.

[2] E. P. D. Freitas, T. Heimfarth, I. F. Netto, C. E. Lino, C. E. Pereira, A. M. Ferreira, F. R. Wagner, and T. Larsson, "UAV relay network to support WSN connectivity," 2010 International Congress on Ultra Modern Telecommunications and Control Systems and Workshops, pp. 309-314, 2010.

[3] A. Agogino, C. HolmesParker, and K. Tumer, "Evolving large scale UAV communication system," Proceedings of the fourteenth international conference on Genetic and evolutionary computation conference - GECCO '12, pp. 1023-1300, 2012.

[4] S. Kim, H. Oh, J. Suk, and A. Tsourdos, "Coordinated trajectory planning for efficient communication relay using multiple UAVs," Control Engineering Practice, vol. 29, pp. 42-49, 2014.

[5] Z. Han, A. L. Swindlehurst, and K. J. R. Liu, "Optimization of MANET connectivity via smart deployment / movement of unmanned air vehicles," IEEE Transactions on Vehicular Technology, vol. 58, no. 7, pp. 3533-3546, 2009.

[6] P. Ladosz, H. Oh, and W.-H. Chen, "Trajectory planning for communication relay unmanned aerial vehicles in urban dynamic environments," Journal of Intelligent \& Robotic Systems, vol. 89, no. 1, pp. 7-25, Jan 2018.

[7] A. Grancharova, E. I. Grotli, D. T. Ho, and T. A. Johansen, "UAVs Trajectory Planning by Distributed MPC under Radio Communication Path Loss Constraints," Journal of Intelligent and Robotic Systems: Theory and Applications, vol. 79, no. 1, pp. 115-134, 2014.

[8] A. Al-Hourani, S. Kandeepan, and S. Lardner, "Optimal LAP altitude for maximum coverage," IEEE Wireless Communications Letters, vol. 3, no. 6, pp. 569-572, 2014.

[9] C. Dixon and E. W. Frew, "Optimizing cascaded chains of unmanned aircraft acting as communication relays," IEEE Journal on Selected Areas in Communications, vol. 30, no. 5, pp. 883-898, 2012.

[10] A. J. Carfang, N. Wagle, and E. W. Frew, "Improving data ferrying by iteratively learning the radio frequency environment," IEEE International Conference on Intelligent Robots and Systems, pp. 1182-1188, 2014.

[11] P. Ladosz, H. Oh, and W.-H. Chen, "Prediction of air-to-ground communication strength for relay uav trajectory planner in urban environments,' in IEEE/RSJ International Conference on Intelligent Robots and Systems 2017, IROS 2017, 2017.

[12] P. T. De Boer, D. P. Kroese, S. Mannor, and R. Y. Rubinstein, “A tutorial on the cross-entropy method," Annals of Operations Research, vol. 134 no. 1, pp. 19-67, 2005.

[13] ITU-R, "Rec. p.1410-2 propagation data and prediction methods for the design of terrestrial broadband millimetric radio access systems," $P$ Series Radiowave propagation, 2003.

[14] A. Al-Hourani, S. Kandeepan, and A. Jamalipour, "Modeling air-toground path loss for low altitude platforms in urban environments," 2014 IEEE Global Communications Conference, pp. 2898-2904, 2014.

[15] M. Mozaffari, W. Saad, M. Bennis, and M. Debbah, "Efficient deployment of multiple unmanned aerial vehicles for optimal wireless coverage," IEEE Communications Letters, pp. 1-4, 2016.

[16] T. Schwengler, "Wireless \& cellular communications class notes for tlen-5510 fall 2017, chapter 4," http://morse.colorado.edu/ tlen5510/ text/classweb.html, 2017, accessed 19/12/2017.

[17] R. Sedgewick and K. Wayne, "Minimum spanning trees," 2015.

[18] J. B. Kruskal, "On the shortest spanning subtree of a graph and the traveling salesman problem," Proceedings of the American Mathematical Society, vol. 7, no. 1, pp. 48-50, 1956.

[19] S. Kim, H. Oh, and A. Tsourdos, "Nonlinear model predictive coordinated standoff tracking of a moving ground vehicle," Journal of Guidance Control and Dynamics, vol. 36, pp. 557-566, August 2013.

[20] K. Mehrotra and P. R. Mahapatra, "A jerk model for tracking highly maneuvering targets," IEEE Transactions on Aerospace and Electronic Systems, vol. 33, no. 4, pp. 1094-1105, 1997. 